Article

\title{
Preparation and Characterization of Urea Formaldehyde @ Epoxy Resin Microcapsule on Waterborne Wood Coatings
}

\author{
Xiaoxing Yan ${ }^{1,2, *}$, Xingyu Qian ${ }^{2}$ and Yijuan Chang ${ }^{2}$ \\ 1 Co-Innovation Center of Efficient Processing and Utilization of Forest Resources, \\ Nanjing Forestry University, Nanjing 210037, China \\ 2 College of Furnishings and Industrial Design, Nanjing Forestry University, Nanjing 210037, China \\ * Correspondence: yanxiaoxing@nuaa.edu.cn; Tel.: +86-25-8542-7528
}

Received: 30 June 2019; Accepted: 25 July 2019; Published: 28 July 2019

\begin{abstract}
Urea formaldehyde @ epoxy resin microcapsules were prepared by two steps in situ polymerization, and the morphology and composition of microcapsules with different mass ratios of core to wall material were examined by scanning electron microscopy (SEM) and infrared (IR) spectroscopy. The microcapsules were added to the waterborne wood coatings to explore the effect of microcapsules on the properties of coatings and their self-repairing characteristics, and the gloss, hardness, adhesion, and impact resistance of the waterborne wood coatings were investigated. The results showed that when the concentration of microcapsule was $10.0 \%$ and the mass ratio of core to wall material was $0.83: 1$, the waterborne wood coatings had a good comprehensive performance and less negative impact. In the scratch test, the waterborne wood coatings with a concentration of $10.0 \%$ microcapsule had good repair ability. The possibility of applying the self-repairing technology of microcapsule to waterborne wood coatings was discussed through the preparation and performance characterization of self-repairing coatings and the results can become a guideline for the engineering application of waterborne wood coatings.
\end{abstract}

Keywords: microcapsule; waterborne coatings; self-repairing; epoxy resin

\section{Introduction}

Wood, as a traditional material, is widely used in construction, furniture, transportation, railway, and other fields [1]. In currency, renewable resources are becoming very important [2]. Human beings are increasingly aware of the importance of environmental protection, conservation, and sustainable development [3]. Wood, as a renewable and environment-friendly resource, has attracted more and more attention [4]. Coating, as an important means of closing wood vessels and improving the stability of wood use, has also been paid more and more attention. Compared with the traditional solvent-based coating, such as polyurethane and nitro [5], the waterborne coatings have the characteristics of low viscosity, low toxicity, anti-flow hang [6], fast drying speed [7], good color, good flexibility, good water resistance, abrasion resistance, aging resistance, and yellowing resistance [8], so it is the favorite of many consumers [9]. However, during the use of waterborne wood coatings, due to the complex coating process and the wet expansion and shrinkage of the wood, the coating causes cracking and other defects easily [10].

Achievements have been made by many scholars on the research of microcapsule self-repairing technology in coatings [11], but the application of microcapsule self-repairing technology is less in the field of waterborne coatings on wood [12]. If the self-repairing microcapsule can be added without affecting the performance of the waterborne wood coatings when the coatings are damaged and 
microcracks occur, the wall of the capsule can be automatically fractured, and the core material is released [13]. The core material (the healing agent) will be polymerized and directly acted to bond the crack [14], and the utilization and safety of the waterborne wood coatings can be improved. Urea formaldehyde resin is hard, scratch-resistant, acid- and alkali-resistant, cheap, and has certain toughness [15]. The epoxy resin material has the characteristics of compactness, water resistance, high leakage resistance, high strength, advantages of strong adhesion, and simple construction [16]. The urea-formaldehyde resin as wall material of microcapsule has good protective and barrier properties to core epoxy resin and good interfacial compatibility with waterborne wood coatings [17]. Therefore, the preparation of urea-formaldehyde coated epoxy resin microcapsules (urea formaldehyde @ epoxy microcapsules) and the properties of wood coatings formed by waterborne coatings with the addition of microcapsules were characterized and tested in order to establish a foundation for the application of coatings in engineering.

\section{Materials and Methods}

\subsection{Material}

Urea $\left(M_{\mathrm{w}}: 60.06 \mathrm{~g} / \mathrm{mol}\right.$, CAS No.: $\left.57-13-6\right), 37 \%$ formaldehyde solution $\left(M_{\mathrm{w}}: 30.03 \mathrm{~g} / \mathrm{mol}\right.$, CAS No.: 50-00-0), epoxy resin ( $M_{\mathrm{W}}: 375.86 \mathrm{~g} / \mathrm{mol}$, CAS No.: 61788-97-4), sodium dodecyl benzene sulfonate $\left(M_{\mathrm{W}}\right.$ : $348.48 \mathrm{~g} / \mathrm{mol}$, CAS No.: 25155-30-0), citric acid monohydrate $\left(M_{\mathrm{w}}: 210.14 \mathrm{~g} / \mathrm{mol}\right.$, CAS No.: 5949-29-1), anhydrous ethanol $\left(M_{\mathrm{W}}: 46.07 \mathrm{~g} / \mathrm{mol}\right.$, CAS No.: 64-17-5), and triethanolamine $\left(M_{\mathrm{W}}: 149.19 \mathrm{~g} / \mathrm{mol}\right.$, CAS No.: 102-71-6) were supplied by Xilong Chemical Co., Ltd., Guangzhou, China. Fraxinus mandshurica Rupr veneered panels (uniform material color, $50 \mathrm{~mm} \times 40 \mathrm{~mm} \times 5 \mathrm{~mm}, 300$ pieces, after ordinary mechanical sanding) were supplied by Yihua Lifestyle Technology Co. Ltd., Shantou, China. The veneer was glued onto the medium-density fiberboard and the thickness of veneer was $1 \mathrm{~mm}$. The studied wood samples have a radial sawn wood texture. Waterborne coatings were supplied by Nippon Paint Co. Ltd., Shanghai, China. Waterborne coatings consisted of acrylic copolymers supported by water, dipropylene glycol methyl ether, and dipropylene glycol butyl ether, and the content was $90.0 \%$, $2.0 \%$, and $8.0 \%$, respectively.

\subsection{Preparation of Microcapsules}

The urea formaldehyde resin formed by urea and formaldehyde was selected as the wall material and epoxy resin was selected as the core material. According to the in-situ polymerization, the mass ratio of core to wall material is $0.83: 1,0.75: 1,0.67: 1$, and $0.58: 1$, respectively. A detailed list of the amount of material for microcapsules is shown in Table 1 . The quantity of the core material is 25.0, $22.5,20.0$, and $17.5 \mathrm{~g}$, respectively, while the quality of the wall material was constant. Four kinds of urea-formaldehyde@ epoxy microcapsules with different mass ratios of the core to wall materials were prepared. The mass ratio of core to wall material is $0.83: 1$ and the preparation process is as follows:

Preparation of urea formaldehyde resin: The mixture of $20.0 \mathrm{~g}$ urea and $34.0 \mathrm{~g} \mathrm{37 \%}$ formaldehyde solution was added to the beaker according to the mass ratio of 1:1.7. The mixture was well-mixed, and the $\mathrm{pH}$ value of the solution was adjusted to $8.0-9.0$ by slowly adding triethanolamine. The mixture was continuously stirred for $1 \mathrm{~h}$ at the constant temperature water bath at $70^{\circ} \mathrm{C}$, and the wall material solution was prepared and cooled at room temperature.

Preparation of core material epoxy resin: $1.95 \mathrm{~g}$ sodium dodecyl benzene sulfonate white powder was added into $193.05 \mathrm{~g}$ deionized water with stirring to complete dissolving, and $1.0 \%$ of sodium dodecyl benzene sulfonate water solution was obtained. $25.0 \mathrm{~g}$ epoxy resin was added to the solution, and the magnetic stirring rotor was put into the solution. The mixture was stirred at the speed condition of $1200 \mathrm{r} / \mathrm{min}$ for $30 \mathrm{~min}$ under water bath of $60^{\circ} \mathrm{C}$, and the stable core material emulsion was obtained.

Microencapsulation: when the rotational speed was set to $300 \mathrm{r} / \mathrm{min}$, the cooling wall material was added into the core emulsion and then the citric acid monohydrate was added to completely dissolve, and the $\mathrm{pH}$ value was adjusted to 2.5-3.0. The system was reacted for $3 \mathrm{~h}$ at $70^{\circ} \mathrm{C}$. After a 
few deionized water and anhydrous ethanol flushing, the products were put into an oven and dried at $80^{\circ} \mathrm{C}$ for $4 \mathrm{~h}$. The final white powder was the required microcapsule. The preparation method of the mass ratio of core to wall material of 0.75:1, 0.67:1, and 0.58:1 was the same as the above.

Table 1. A detailed list of the amount of material for microcapsules.

\begin{tabular}{ccccccc}
\hline Sample & $\begin{array}{c}\text { Sodium Dodecyl } \\
\text { Benzene } \\
\text { Sulfonate/g }\end{array}$ & $\begin{array}{c}\text { Deionized } \\
\text { Water/g }\end{array}$ & $\begin{array}{c}\text { Epoxy } \\
\text { Resin/g }\end{array}$ & Urea/g & $\begin{array}{c}\text { Formaldehyde } \\
\text { Solution/g }\end{array}$ & $\begin{array}{c}\text { Mass Ratio of } \\
\text { Core to Wall } \\
\text { Material }\end{array}$ \\
\hline 1 & 1.95 & 193.05 & 25.0 & 20.0 & 34.0 & $0.83: 1$ \\
2 & 1.76 & 174.24 & 22.5 & 20.0 & 34.0 & $0.75: 1$ \\
3 & 1.56 & 154.44 & 20.0 & 20.0 & 34.0 & $0.67: 1$ \\
4 & 1.37 & 135.63 & 17.5 & 20.0 & 34.0 & $0.58: 1$ \\
\hline
\end{tabular}

\subsection{Preparation of Coatings}

First, the Fraxinus mandshurica Rupr veneer was placed at room temperature and $50.0 \% \pm 5.0 \%$ relative humidity for one week to achieve a balanced moisture content of the substrate. The putty was used to fill the vessels of the base material. Forty grams of talc powder were added to $100.0 \mathrm{~g}$ water and mixed evenly to obtain the putty, which was coated evenly on the substrate. After the putty was dry, 600 grit sandpaper was used to sand the Fraxinus mandshurica Rupr veneer along the texture, then the dry cloth was used to wipe off the dust. The acrylic copolymer waterborne coating was coated on the veneer using a SZQ tetrahedral fabricator (Tianjin Jinghai Science and Technology Testing Machinery Factory, Tianjin, China). The veneer was fixed on the platform, then the waterborne coating was poured in front of the fabricator. The required coating thickness can be obtained by grasping both ends of the fabricator by hand and sliding at a speed of $150 \mathrm{~mm} / \mathrm{s}$. After 30 min of natural drying, 1000 grit sandpaper was used to sand the coating and a dry cloth was used to wipe off the dust. After repeating the process three times, the primer processing of substrate was complete. Then, $5.0 \mathrm{~g}$ prepared microcapsules (the mass ratio of core to wall material of $0.83: 1$ ) were added to $95.0 \mathrm{~g}$ waterborne coatings and mixed evenly, forming the coatings with $5.0 \%$ concentration of microcapsules. The waterborne coatings with $10.0 \%, 15.0 \%, 20.0 \%$, and $25.0 \%$ concentration of microcapsules were also prepared. The formulations are shown in Table 2. After the three-layer primer was dried, the waterborne coatings with different concentrations of microcapsules were prepared using the SZQ tetrahedral fabricator as the topcoat. The coating was dried under the environment of $40{ }^{\circ} \mathrm{C}$ for $4 \mathrm{~h}$ and then sanded using 1000 grit sandpaper, and a dry cloth was used to wipe off the dust. The thickness of the dry waterborne coating was about $60 \mu \mathrm{m}$. The waterborne coatings without microcapsule were prepared according to the same process and the coating thickness was about $60 \mu \mathrm{m}$ for comparison. Forty samples were prepared for each microcapsules ration. The waterborne coatings with and without microcapsules (the mass ratio of core to wall material of $0.83: 1,0.75: 1,0.67: 1$, and $0.58: 1$ ) were prepared onto glass substrates according to the same process and the coating thickness was about $60 \mu \mathrm{m}$ for tensile test measurement.

Table 2. Composition of waterborne coatings.

\begin{tabular}{cccc}
\hline Sample & Concentration (\%) & Microcapsules $(\mathbf{g})$ & Waterborne Coatings $(\mathbf{g})$ \\
\hline 1 & 0 & 0 & 100.0 \\
2 & 5.0 & 5.0 & 95.0 \\
3 & 10.0 & 10.0 & 90.0 \\
4 & 15.0 & 15.0 & 85.0 \\
5 & 20.0 & 20.0 & 80.0 \\
6 & 25.0 & 25.0 & 75.0 \\
\hline
\end{tabular}




\subsection{Test and Characterization}

The microstructure of the microcapsules was analyzed using a Quanta 200 environment scanning electron microscope (SEM), FEI Company, Hillsboro, OR, USA and L2800 Biomicroscope, Guangzhou Liss Optical Instrument Co., Ltd., Guangzhou, China. The components of the microcapsules were analyzed using VERTEX 80V infrared spectrum analyzer, Germany BRUKER Co., Ltd., Karlsruhe, Germany. The strength of the waterborne coatings on Fraxinus mandshurica Rupr veneer was measured by a QCJ impactor. The location of the impact was in the early wood. In the impact test, a $1.0 \mathrm{~kg}$ ball fell on the coating surface, and the maximum distance between the ball and the coating was $50.0 \mathrm{~cm}$. After the ball fell on the coating surface, the damage of the coating was observed. When the coating was not destroyed, the maximum drop height of the ball was the impact strength. A QFZ-II coating adhesion tester was used to test the adhesion of the coating on the Fraxinus mandshurica Rupr veneer. When measuring, the tip of the rotary needle contacts the coating, and the handle shakes clockwise. The rotational speed is in the range of $80-100 \mathrm{r} / \mathrm{min}$, and the scratch length of the circular roller is $7.5 \pm 0.5 \mathrm{~mm}$. At the end of the adhesion test, the damage of the coating was observed with a magnifying glass. The marks $0,1,2,3,4,5,6$, and 7 on the top of the coatings are the eight grades of damage. Grade 0 has the strongest adhesion and grade 7 has the weakest adhesion. Impactor and adhesion tester were both produced by Tianjin Jingkelian Material Testing Machine Co. Ltd., Tianjin, China. The $6 \mathrm{H}-6 \mathrm{~B}$ pencil test was used to test the hardness of the coating on the Fraxinus mandshurica Rupr veneer. A BGD512-60 gloss meter produced by Suzhou Essen Instrument Equipment Co. Ltd., Suzhou, China, was used to measure the gloss of the waterborne coatings on the Fraxinus mandshurica Rupr veneer. The elongation at break of the coatings was measured by the Model AG-IC100KN precision electronic universal capability experiment machine (Shimadzu Co., Ltd., Kyoto, Japan) and TRview X optical displacement meter (Shimadzu Co., Ltd., Kyoto, Japan). After peeling off the coating on the glass substrate, the coatings were prepared into thin strips, and then the two ends of the coating were clamped with fixtures to ensure that it would not slide. The coating deformed at $0.12 \mathrm{~mm} / \mathrm{min}$ tensile speed and was destroyed under a certain longitudinal load. The elongation at break was calculated according to the displacement length of the coating at break and initial length of the coating before stretching. All the measurements were repeated at least four times with an error of less than $5.0 \%$.

\section{Results and Discussion}

\subsection{Morphological Analysis of Microcapsules}

In Figure 1, four SEM images of (A), (B), (C), and (D) correspond to microcapsules of core to wall mass ratio of $0.83: 1,0.75: 1,0.67: 1$, and $0.58: 1$. The pictures showed that the microcapsules with spherical morphology, relatively uniform particle size, and higher yield were successfully prepared by in situ composite methods [18]. The average size of microcapsules is $3-5 \mu \mathrm{m}$. The change in the mass ratio of the core to wall has little effect on the size of microcapsules. By comparing (A), (B), (C), and (D) using four electron micrographs, it is obvious that the microcapsule morphology of Figure 1A, a round ball with uniform particle size of the microcapsules, was the best. It is obvious that the microcapsules are very complete, with almost no breakage and no serious agglomeration. In addition, a smooth surface was observed. SEM showed that the microcapsule with core to wall material mass ratio of $0.83: 1$ was the best. 

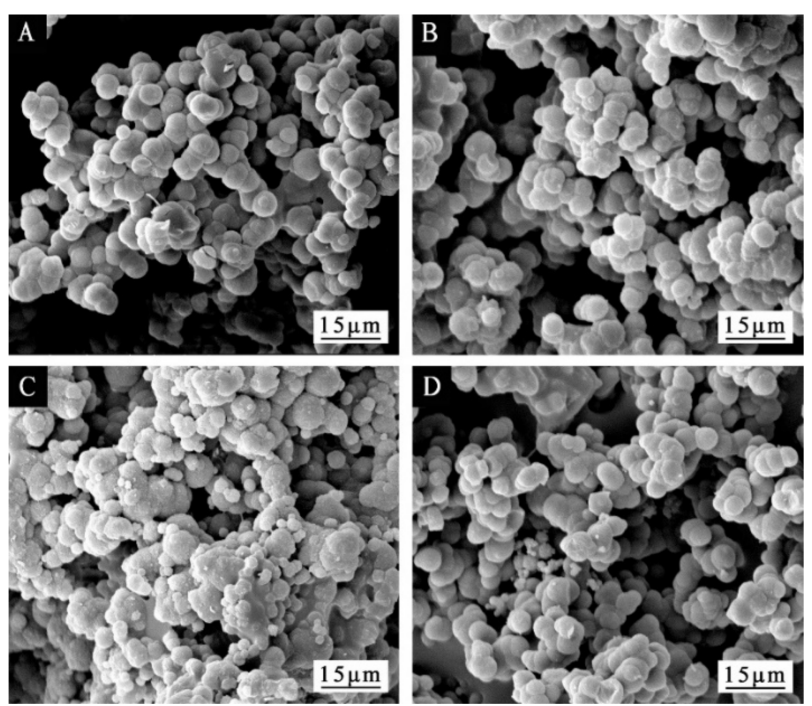

Figure 1. SEM of microcapsule with the core to wall mass ratio of (A) $0.83: 1$, (B) $0.75: 1,(\mathbf{C})$ 0.67:1, and (D) $0.58: 1$.

The elongation at break of the coatings with different core to wall mass ratios is shown in Figure 2. When the core to wall mass ratio kept constant, the elongation at break increased first and then decreased with the increase of microcapsule concentration. When the concentration of microcapsules increased from 0 to $10.0 \%$, the elongation at break of coatings with core to wall mass ratios of 0.83:1, $0.75: 1,0.67: 1$, and $0.58: 1$ increased from $2.67 \%$ to $35.0 \%, 20.2 \%, 15.8 \%$, and $14.7 \%$, respectively, which may have been due to the increase of toughness of the coating [18]. When the concentration of microcapsules increased from $10.0 \%$ to $25.0 \%$, the elongation at break of microcapsules with core to wall mass ratios of $0.83: 1,0.75: 1,0.67: 1$, and $0.58: 1$ decreased gradually. It may be that the high microcapsules concentration leads to the agglomerated particles, which decreased of elongation at break [18]. When the mass ratio of core to wall of microcapsule was $0.83: 1$, the elongation at break of the coating was higher and the mechanical properties were better. Therefore, microcapsules with the core to wall mass ratio of 0.83:1 were added to waterborne coatings to prepare wood coatings.

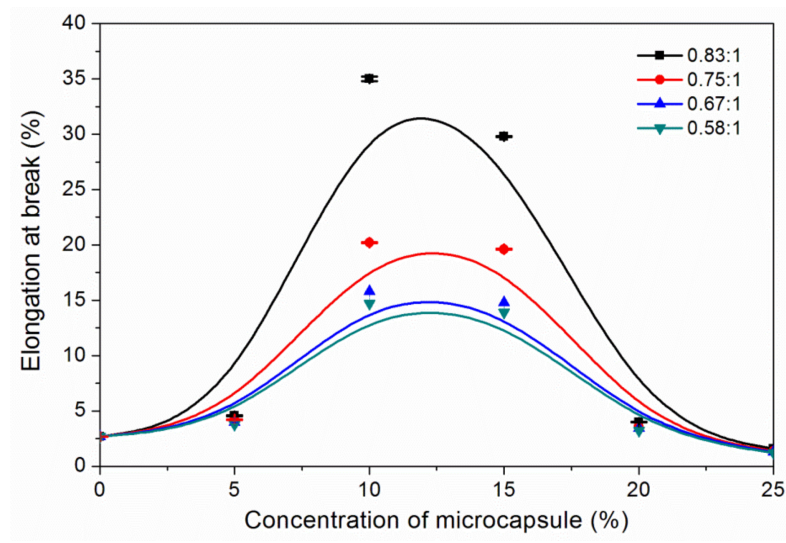

Figure 2. Effect of microcapsules with different core to wall mass ratios on elongation at break of coatings.

\subsection{Chemical Composition Analysis}

Figure 3 is an infrared spectrogram of urea formaldehyde @ epoxy resin microcapsule prepared by the core to wall materials with a mass ratio of 0.83:1. The attribution of peaks was shown in Table 3 . From Figure 3, it is shown that special absorption peaks appear around 3360, 2966, 1645, and $1556 \mathrm{~cm}^{-1}$, which are the characteristic absorption of $\mathrm{N}-\mathrm{H}, \mathrm{C}-\mathrm{H}, \mathrm{C}=\mathrm{O}$, and $\mathrm{C}-\mathrm{N}$, respectively [19]. The functional 
groups of urea formaldehyde resin formed by urea and formaldehyde were characterized. It was confirmed that the corresponding wall materials of urea formaldehyde resin were generated in the prepared microcapsules. The characteristic absorption of $1247 \mathrm{~cm}^{-1}$ represents the symmetrical vibration absorption of the epoxy group [20], indicating that the epoxy resin has already existed in the microcapsules and the chemical structure has not been destroyed. Therefore, for the microcapsules, epoxy resin was successfully coated with urea formaldehyde resin.

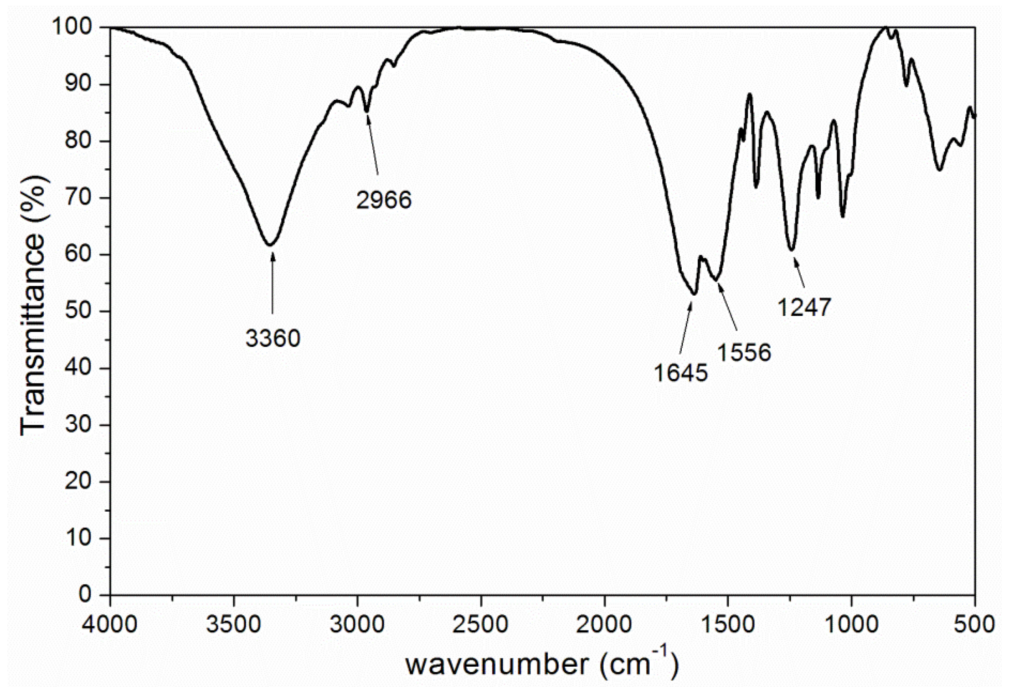

Figure 3. Infrared spectroscopy of microcapsules with the mass ratio of core to wall materials: $0.83: 1$.

Table 3. Assignment of peaks.

\begin{tabular}{cc}
\hline Peak $\left(\mathbf{c m}^{-\mathbf{1}}\right)$ & Assignment \\
\hline 3360 & absorption of $\mathrm{N}-\mathrm{H}$ \\
2966 & absorption of $\mathrm{C}-\mathrm{H}$ \\
1645,1724 & absorption of $\mathrm{C}=\mathrm{O}$ \\
1556 & absorption of $\mathrm{C}-\mathrm{N}$ \\
1247 & symmetrical vibration absorption of epoxy group \\
810 & absorption of $\mathrm{C}=\mathrm{C}-\mathrm{H}$ \\
1150 & absorption of $\mathrm{C}-\mathrm{O}-\mathrm{C}$ \\
\hline
\end{tabular}

Figure 4 is the infrared spectrum of waterborne coating with the core to wall mass ratio of 0.83:1 and different addition amount. From the graph, we can see that $\mathrm{C}=\mathrm{C}-\mathrm{H}$ has been absorbed near $810 \mathrm{~cm}^{-1}$. Near the $1150 \mathrm{~cm}^{-1}$, the absorption of $\mathrm{C}-\mathrm{O}-\mathrm{C}$ appeared. $1724 \mathrm{~cm}^{-1}$ represents the absorption of $\mathrm{C}=\mathrm{O}$. The stretching vibration of $\mathrm{C}-\mathrm{H}$ appeared at $2966 \mathrm{~cm}^{-1}$ [21]. The waterborne coating with microcapsules added showed the same infrared spectrum (Figure 4 and Table 3). The characteristic peaks of urea formaldehyde resin and epoxy resin appeared, indicating that the components of microcapsules existed and were not damaged in waterborne coating [22]. 


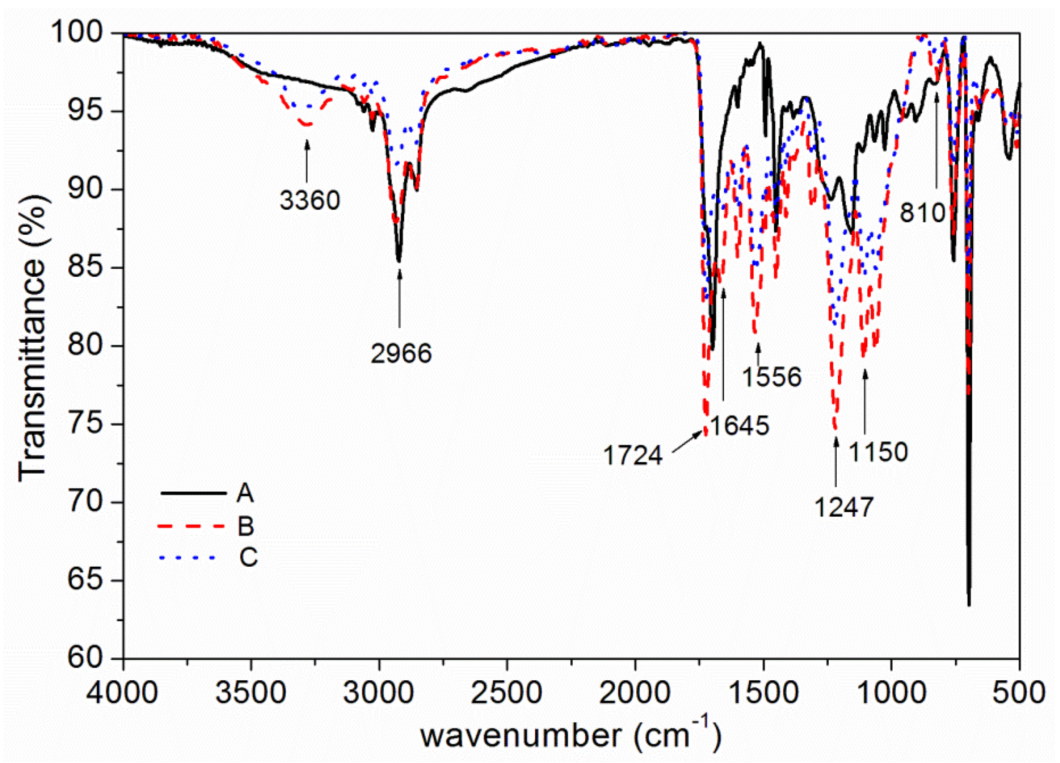

Figure 4. The influence of the mass ratio and the concentration of microcapsule on the waterborne coatings. (A) waterborne coatings. The mass ratio of core to wall materials was $0.83: 1$, the concentration of microcapsule was $5.0 \%(\mathbf{B})$ and $25.0 \%(\mathbf{C})$, respectively.

\subsection{The Properties of Waterborne Coatings after Adding Microcapsules}

The microcapsules (the mass ratio of core to wall material of $0.83: 1$ ) were added to the waterborne coatings with the addition of $5.0 \%, 10.0 \%, 15.0 \%, 20.0 \%$, and $25.0 \%$, respectively, and the gloss, hardness, adhesion, and impact resistance of the coating were evaluated. As shown in Figure 5, the addition of microcapsules can reduce the gloss of the waterborne coatings [23]. The gloss of the coating surface is negatively correlated with the increase of microcapsule addition, and when the amount of addition increased from 0 to $25.0 \%$, the gloss decreased from $81.3 \%$ to $0.4 \%$. Because the prepared microcapsule is the white powder, adding the coating will change the original gloss of the coating and reduce the gloss effect of the waterborne coatings [24]. Furthermore, the roughness of the coating surface increased due to the addition of the microcapsules (Figure 6), which negatively affects the gloss.

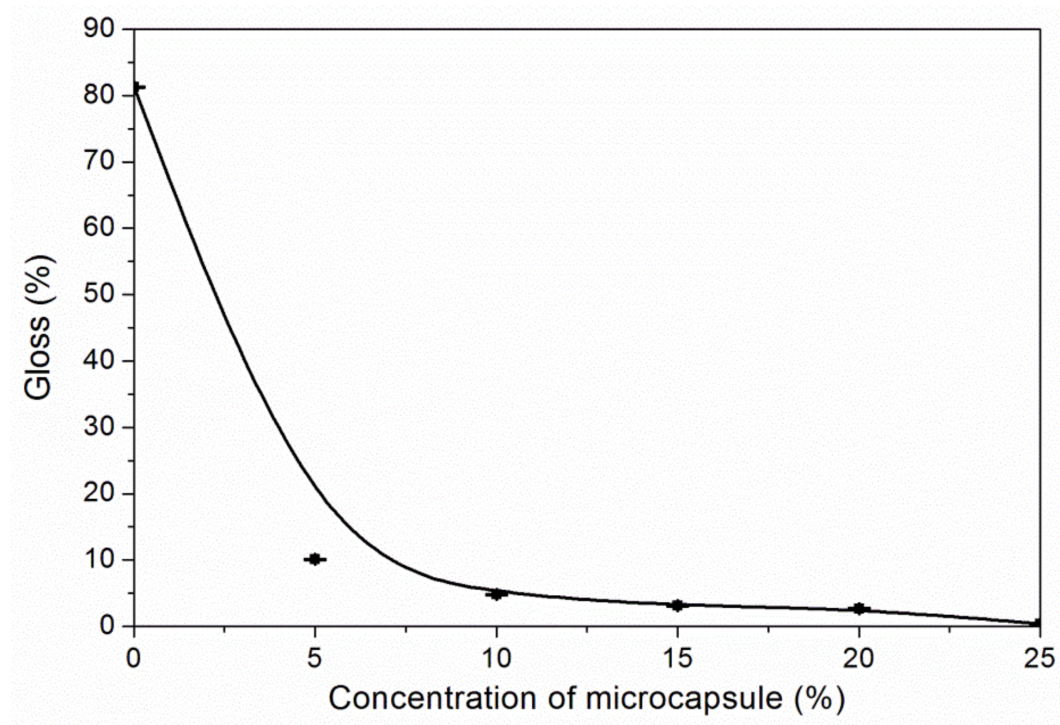

Figure 5. Effect of different concentration of microcapsules on the gloss of waterborne coatings. 


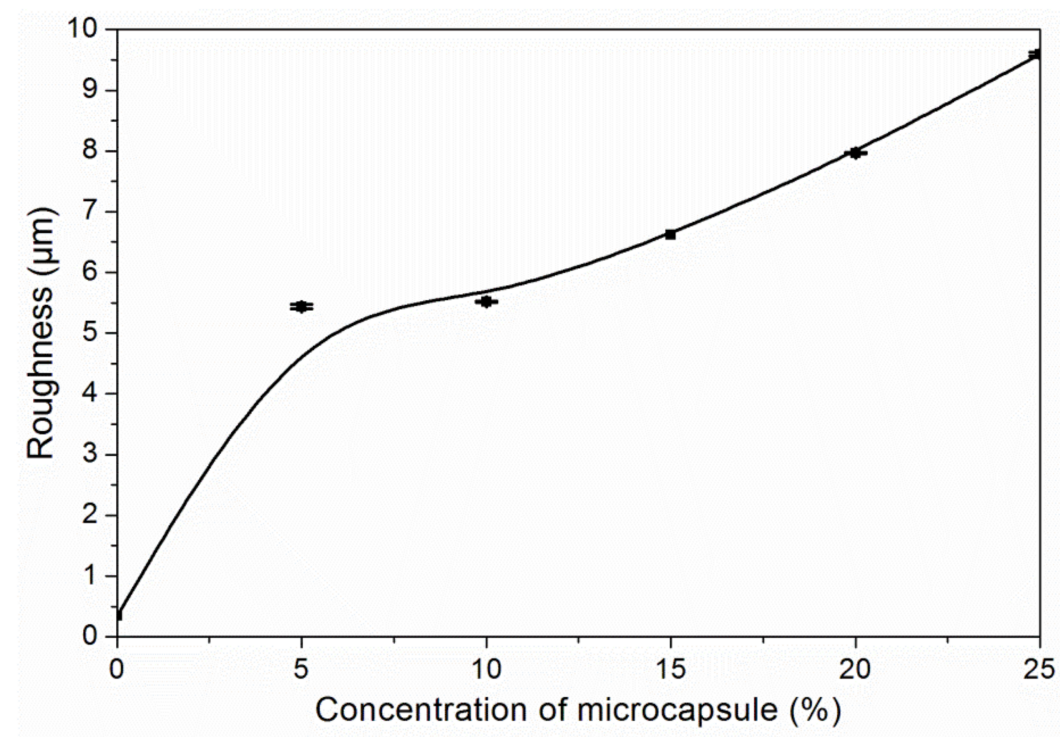

Figure 6. Effect of different concentration of microcapsules on the roughness of waterborne coatings.

As shown in Figure 7, the addition of microcapsules has a significant effect on the hardness of the waterborne coatings. In a certain range, the higher the number of microcapsules, the higher the hardness of the coating. When the addition of microcapsules increased from 0 to $10.0 \%$, the hardness increased from $\mathrm{H}$ to $5 \mathrm{H}$. When the addition of microcapsules increased from $10.0 \%$ to $25.0 \%$, the hardness of the waterborne coatings tended to be $5 \mathrm{H}$, so the best addition of microcapsules was $10.0 \%$. At this time, the additional concentration of the microcapsules to the waterborne coatings was the least and the hardness of the coating was the best.

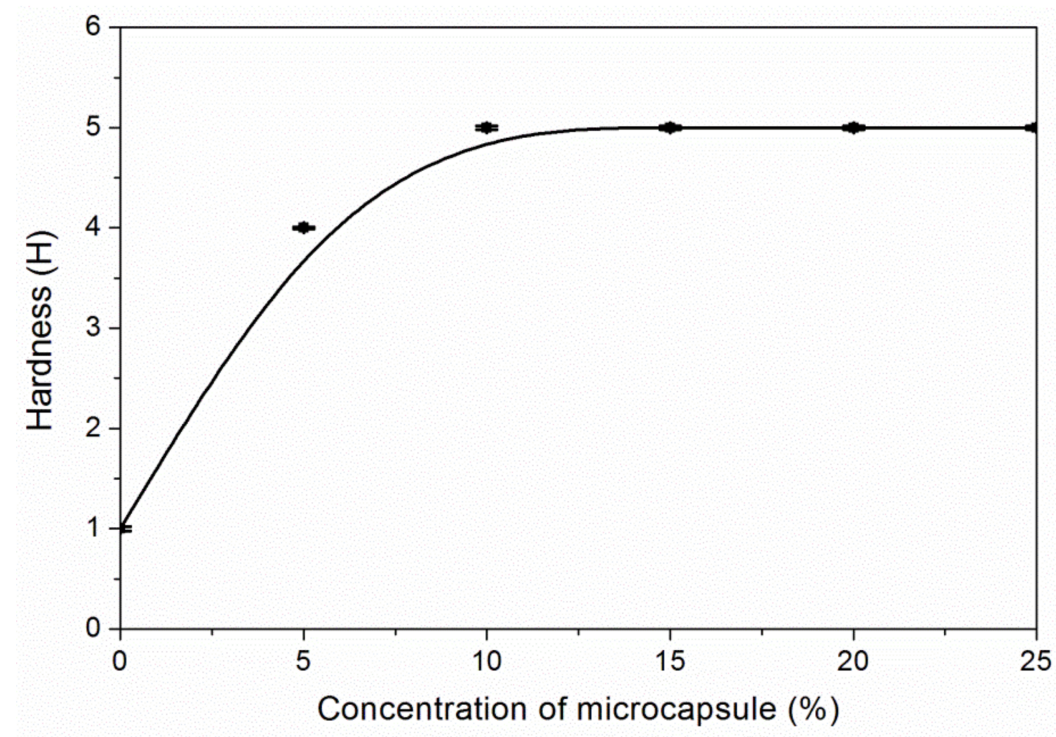

Figure 7. Effect of microencapsulation addition on the hardness of waterborne coatings.

When the addition of microcapsules increased from 0 to $25.0 \%$, the adhesion of the coating did not change, and the grade was 3 (Figure 8). It may be due to the small agglomeration of the microcapsules and the more uniform dispersion in the coating, which did not affect the adhesion of the coating [25]. 


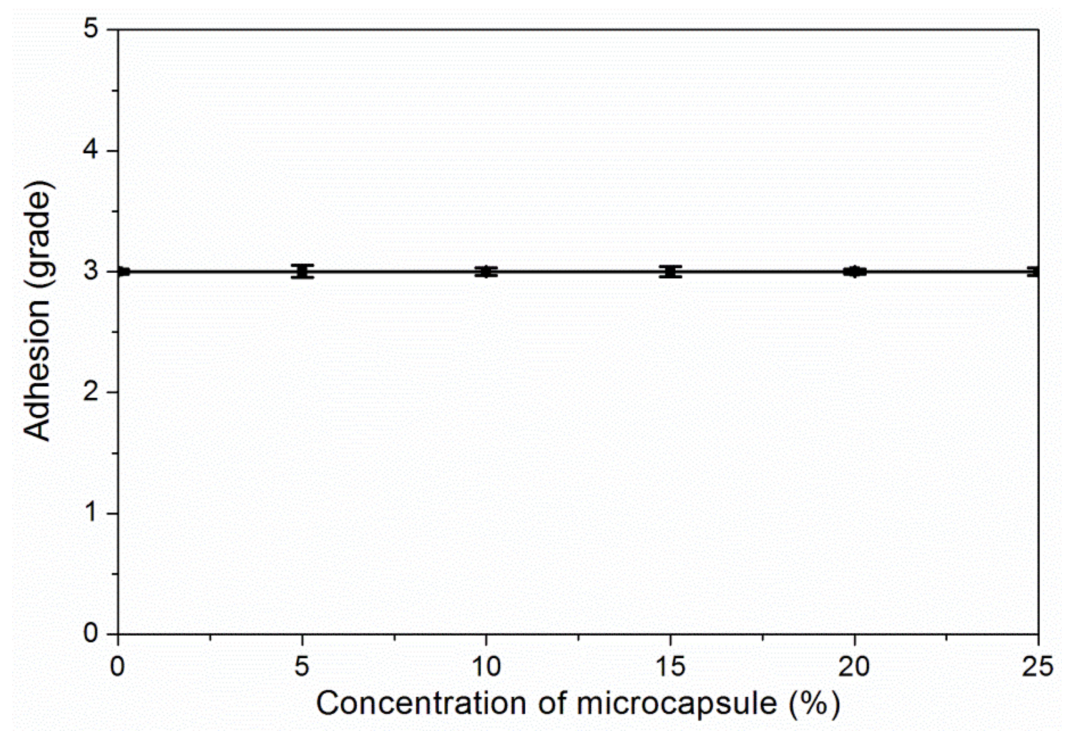

Figure 8. Effect of microencapsulation addition on the adhesion of waterborne coatings.

As shown in Figure 9, the concentration of microcapsules had an impact on the impact resistance. When the addition of microcapsules increased from 0 to $5.0 \%$, the impact strength of the waterborne coatings increased from 5 to $20 \mathrm{~kg} \cdot \mathrm{cm}$. When the addition of microcapsules increased from $5.0 \%$ to $15.0 \%$, the impact resistance of the coating did not change significantly. When the addition of microcapsules increased from $15.0 \%$ to $20.0 \%$, the impact strength of the waterborne coatings increased from 20 to $25 \mathrm{~kg} \cdot \mathrm{cm}$. Although the increasing trend of impact resistance was obvious, the increase in amplitude was lower. When the addition amount increased from $20.0 \%$ to $25.0 \%$, the impact resistance of waterborne coatings was almost unaffected. Therefore, when the concentration of microcapsule was $20.0 \%$ to $25.0 \%$, the waterborne coatings had the strongest impact resistance. To sum up, when the amount of microcapsule added at $10.0 \%$, it has little effect on the original properties of waterborne coatings, which had a high gloss, greater adhesion and impact resistance, and the comprehensive performance was the best.

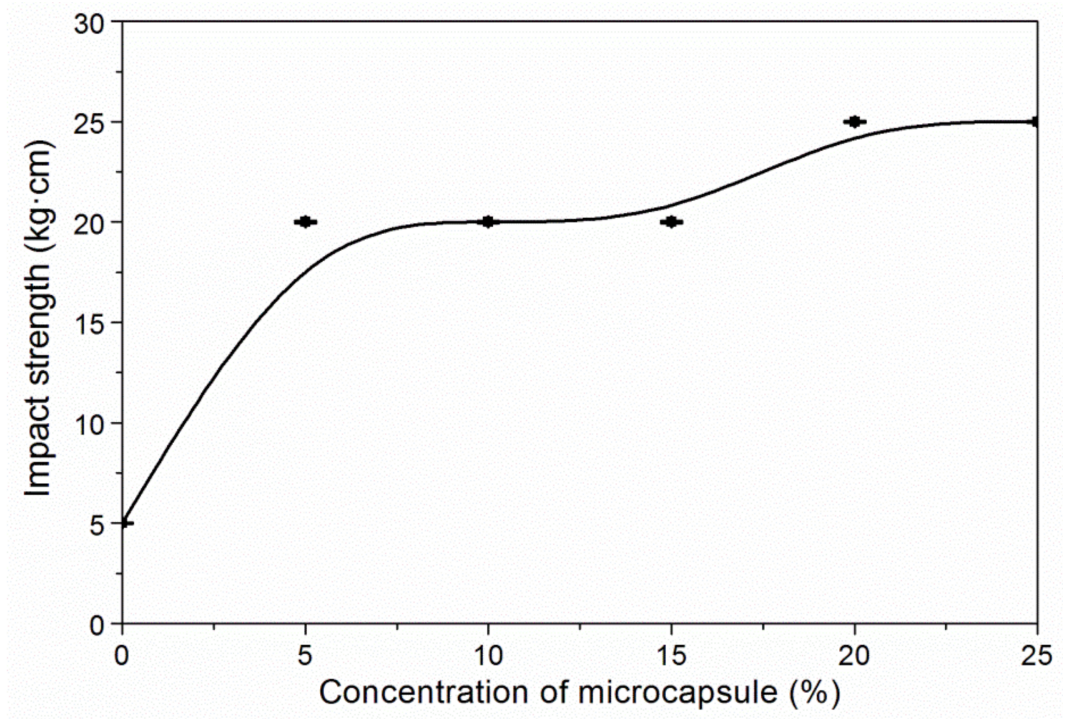

Figure 9. Effect of different concentration of microcapsules on impact resistance of waterborne coatings. 


\subsection{Repair Test of Waterborne Coating with the Microcapsule}

In order to further explore the effects of microcapsules on the damage and microstructure of the coating, it is necessary to destroy the coating before observing the repairing effect of microcapsules. In this experiment, the coating was heated at a constant temperature using the oven to accelerate the damage of the coating, so as to simulate the damage of the coating in daily use. Samples of waterborne coatings containing microcapsules with different mass fractions (Figure 10) were put into the oven to heat and observe the change of coating. First, the samples were placed in the oven and dried for $1.0 \mathrm{~h}$ at $80^{\circ} \mathrm{C}$, and no obvious change was found in the film. When the temperature was increased to $140{ }^{\circ} \mathrm{C}$ and the heating time was prolonged to $4.5 \mathrm{~h}$, bubbling cracking was found in the film, which proved that the film was damaged. The samples were taken out and cooled to room temperature. SEM was used to observe the damage of the coating.
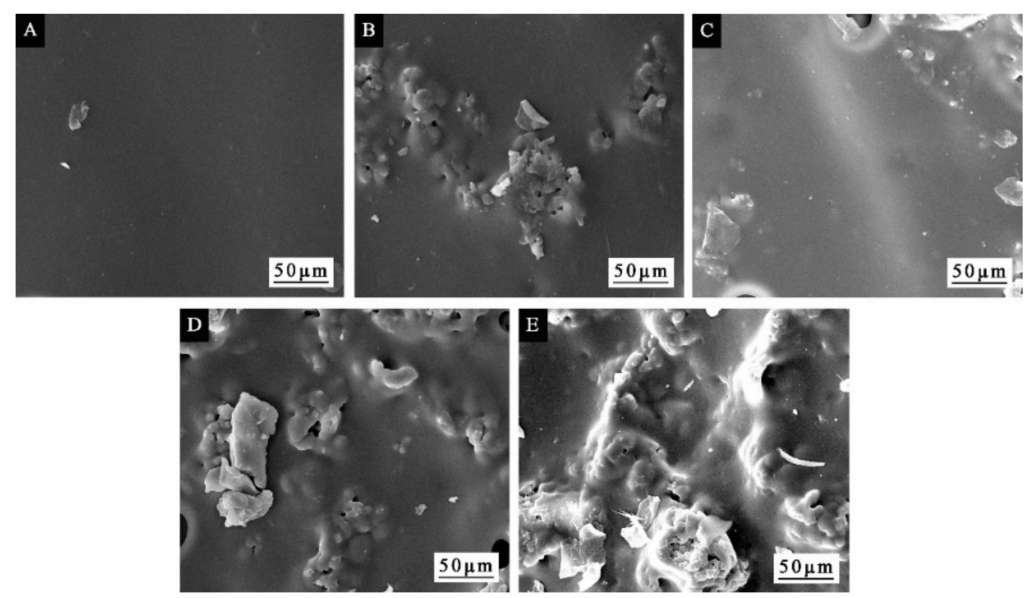

Figure 10. SEM of undamaged waterborne coatings with different concentration of microcapsule added:

(A) 0 , (B) 5.0\%, (C) 10.0\%, (D) 15.0\%, and (E) $20.0 \%$.

Figure 11 showed the damage of the coating as the mass fraction of microcapsules increased. When the mass fraction of microcapsules increased, the number of coating bubbles increased but the area of single bubble gradually decreased, indicating that microcapsules had a certain effect on reducing the degree of damage to the coating. When the mass fraction of microcapsules was $10.0 \%$, the average size of bubbles was relatively small, indicating that the addition of microcapsules can prolong the time needed for the film to be damaged.

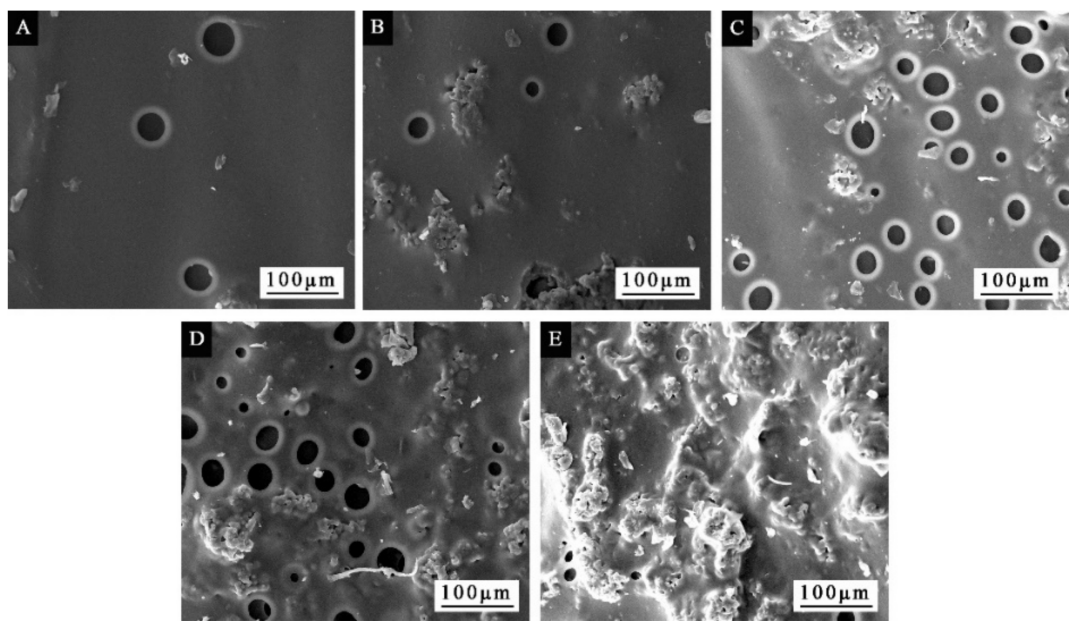

Figure 11. SEM of damaged waterborne coatings with different concentration of microcapsule added: (A) 0 , (B) $5.0 \%$, (C) $10.0 \%$, (D) $15.0 \%$, and (E) $20.0 \%$. 
Then, $10.0 \%$ microcapsules were added to the waterborne coatings for scratch test [26]. The operation method is as follows: A scratch is made in the middle of the coating with a cutter for subsequent observation under the biomicroscope. The results were as shown in Figure 12. Figure 12A is a microscope picture taken after a scratch. Figure 12B is a microscope taken for the same location a week later, and the red frame is the scratch position of the coating. The scratches at the red frame at Figure 12B showed that the scratches of the coating basically disappear after a week, indicating that the microcapsules in the coating were damaged by the scratched external force, the epoxy resin from the core material flows out, and the scratches were filled and repaired. Therefore, it can be proved that the prepared urea formaldehyde @ epoxy microcapsule has a certain repairability for microcracks of the waterborne coating and has good repair performance when the amount of adding concentration in the waterborne coating was $10.0 \%$. It can be seen that when the self-repairing coating is prepared by adding urea formaldehyde @ epoxy resin microcapsules, the cracking of the coating produces microcracks, and the epoxy resin of the core can exist steadily before the capsule rupture, and the thickness and strength of the wall are more appropriate and have a good tightness, and the core material cannot leak for a long time.

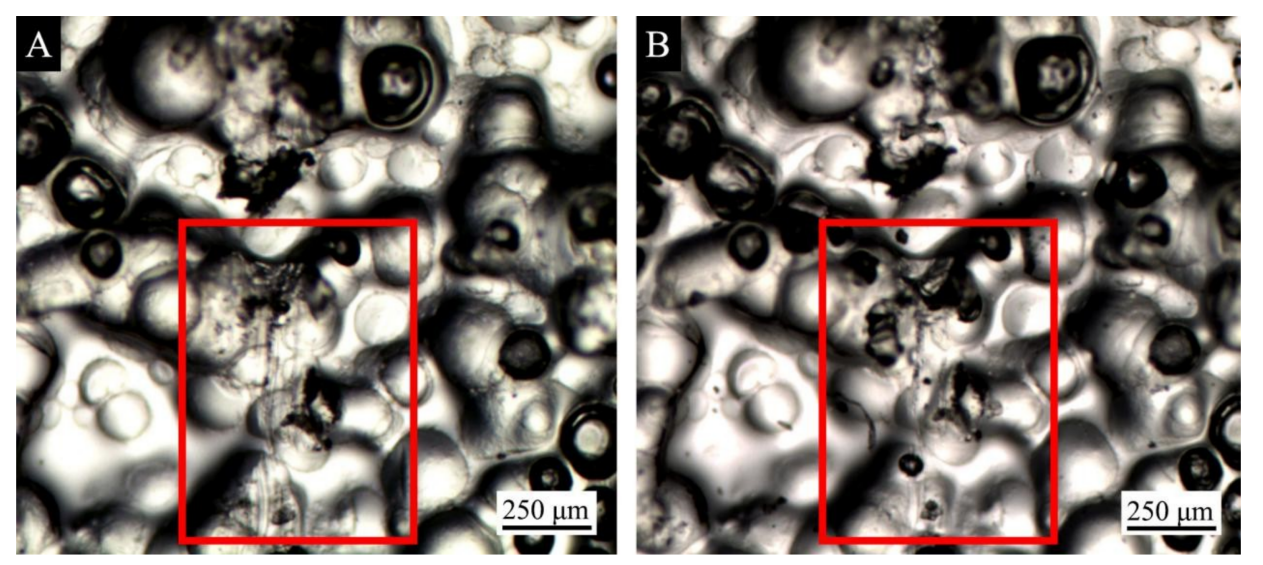

Figure 12. Comparison of a coating before and after microcapsule repair: (A) Before repair and (B) after repair.

\section{Conclusions}

The preparation of urea formaldehyde @ epoxy microcapsule and the effect of adding microcapsules to waterborne coatings on the properties of the coatings were investigated. The results showed that when the core to wall mass ratio was $0.83: 1$, the microcapsules with good morphology, uniform particle size, and lower degree of damage can be prepared. It is preliminarily proved that the prepared microcapsules have self-repairing abilities for the microcracks produced by the damage of waterborne coatings. The gloss of waterborne coatings is the most suitable when the addition of microcapsules is $10.0 \%$. When the microcapsule addition amount was $10.0 \%$, the hardness of the coating was the best, which was $5 \mathrm{H}$, and at this time, the least amount was added. The maximum impact resistance is $25 \mathrm{~kg} \cdot \mathrm{cm}$ when the microcapsule concentration was $20.0 \%-25.0 \%$. The microcapsule addition amount has almost no effect on the adhesion of the coating. Through comprehensive consideration, it was proven that when the microcapsules added to the waterborne coating is $10.0 \%$, it will not reduce the performance of the coating and there is enough repair agent to repair the damage. Therefore, the performance of the coating is more suitable, and the excellent hardness, impact resistance, adhesion, and good repair performance can be obtained.

Author Contributions: Conceptualization, Methodology, Validation, Resources, Data Curation, Writing-Original Draft Preparation, and Supervision, X.Y.; Formal Analysis, X.Q. and Y.C.; Investigation and Writing-Review and Editing, X.Q. 
Funding: This project was partly supported by the Natural Science Foundation of Jiangsu Province (BK20150887) and Youth Science and Technology Innovation Fund of Nanjing Forestry University (CX2016018).

Conflicts of Interest: The authors declare no conflict of interest.

\section{References}

1. Xu, W.; Wu, Z.H.; Zhang, J.L. Compressive creep and recovery behaviors of seat cushions in upholstered furniture. Wood Fiber Sci. 2015, 47, 431-444.

2. Gu, Y.T.; Bian, H.Y.; Wei, L.Q.; Wang, R.B. Enhancement of hydrotropic fractionation of poplar wood using autohydrolysis and disk refining pretreatment: morphology and overall chemical characterization. Polymers 2019, 11, 685. [CrossRef] [PubMed]

3. Xiong, X.Q.; Bao, Y.L.; Guo, W.J.; Fang, L.; Wu, Z.H. Preparation and application of high performance corn starch glue in straw decorative panel. Wood Fiber Sci. 2018, 50, 88-95.

4. Wu, J.M.; Wu, Y.; Yang, F.; Tang, C.Y.; Huang, Q.T.; Zhang, J.L. Impact of delignification on morphological, optical and mechanical properties of transparent wood. Compos. Part A: Appl. Sci. Manuf. 2019, 117, 324-331. [CrossRef]

5. Liu, H.J.; Li, C.; Sun, X.S. Soy-oil-based waterborne polyurethane improved wet strength of soy protein adhesives on wood. Int. J. Adhes. Adhes. 2017, 73, 66-74. [CrossRef]

6. Herrera, R.; Muszynska, M.; Krystofiak, T.; Labidi, J. Comparative evaluation of different thermally modified wood samples finishing with UV-curable and waterborne coatings. Appl. Surf. Sci. 2015, 357, 1444-1453. [CrossRef]

7. Yan, X.X.; Qian, X.Y.; Lu, R.; Miyakoshi, T. Synergistic effect of addition of fillers on properties of interior waterborne UV-curing wood coatings. Coatings 2018, 8, 9. [CrossRef]

8. Zhang, S.W.; Yu, A.X.; Song, X.Q.; Liu, X.Y. Synthesis and characterization of waterborne UV-curable polyurethane nanocomposites based on the macromonomer surface modification of colloidal silica. Prog. Org. Coat. 2013, 76, 1032-1039. [CrossRef]

9. Yan, X.X.; Qian, X.Y.; Lu, R.; Miyakoshi, T. Comparison and optimization of reactive dyes and coating performance on Fraxinus mandshurica veneer. Polymers 2018, 10, 1302. [CrossRef]

10. Jang, G.; Seo, S.; Lee, T.S. Electrostatically self-assembled microcapsule composed of conjugated polyelectrolytes and polypeptides for an emission color-changeable assay for trypsin. Sens. Actuators B 2015, 221, 1229-1235. [CrossRef]

11. Liao, H.Z.; Zhang, B.; Huang, L.H.; Ma, D.; Jiao, Z.P.; Xie, Y.S.; Tan, S.Z.; Cai, X. The utilization of carbon nitride to reinforce the mechanical and thermal properties of UV-curable waterborne polyurethane acrylate coatings. Prog. Org. Coat. 2015, 89, 35-41. [CrossRef]

12. Song, J.H.; Cui, X.F.; Liu, Z.; Jin, G.; Liu, E.B.; Zhang, D.; Gao, Z.H. Advanced microcapsules for self-healing conversion coating on magnesium alloy in $\mathrm{Ce}\left(\mathrm{NO}_{3}\right)_{3}$ solution with microcapsules containing $\mathrm{La}\left(\mathrm{NO}_{3}\right)_{3}$. Surf. Coat. Technol. 2016, 307, 500-505. [CrossRef]

13. Guo, W.C.; Jia, Y.; Tian, K.S.; Xu, Z.P.; Jiao, J.; Li, R.F.; Wu, Y.H.; Cao, L.; Wang, H.Y. UV-Triggered self-healing of a single robust $\mathrm{SiO}_{2}$ microcapsule based on cationic polymerization for potential application in aerospace coatings. ACS Appl. Mater. Interfaces 2016, 8, 21046-21054. [CrossRef] [PubMed]

14. Zhang, C.; Wang, H.R.; Zhou, Q.X. Preparation and characterization of microcapsules based self-healing coatings containing epoxy ester as healing agent. Prog. Org. Coat. 2018, 125, 403-410. [CrossRef]

15. Li, W.T.; Zhu, X.J.; Zhao, N.; Jiang, Z.W. Preparation and properties of melamine urea-formaldehyde microcapsules for self-healing of cementitious materials. Materials 2016, 9, 152. [CrossRef] [PubMed]

16. Tripathi, M.; Kumar, D.; Roy, P.K. Microencapsulation of reactive amine by interfacially engineered epoxy microcapsules for smart applications. Iran. Polym. J. 2017, 26, 489-497. [CrossRef]

17. Cai, X.L.; Fu, D.T.; Qu, A.L. Effects of processing conditions on the properties of epoxy resin microcapsule. J. Wuhan Univ. Technol. 2015, 30, 689-694. [CrossRef]

18. Yan, X.X.; Wang, L.; Qian, X.Y. Effect of urea-formaldehyde-coated epoxy microcapsule modification on gloss, toughness and chromatic distortion of acrylic copolymers waterborne coating. Coatings 2019, 9, 239. [CrossRef] 
19. Liu, Q.; Zhang, J.P.; Liu, W.L.; Guo, F.C.; Pei, J.Z.; Zhu, C.Z.; Zhang, W.W. Preparation and characterization of self-healing microcapsules embedding waterborne epoxy resin and curing agent for asphalt materials. Constr. Build. Mater. 2018, 183, 384-394. [CrossRef]

20. Hillewaere, X.K.D.; Teixeira, R.F.A.; Nguyen, L.T.T.; Ramos, J.A.; Rahier, H.; Du Prez, F.E. Autonomous self-healing of epoxy thermosets with thiol-isocyanate chemistry. Adv. Funct. Mater. 2014, 24, 5575-5583. [CrossRef]

21. Sun, D.W.; Zhang, H.; Tang, X.Z.; Yang, J.L. Water resistant reactive microcapsules for self-healing coatings in harsh environments. Polymer 2016, 91, 33-40. [CrossRef]

22. Bolimowski, P.A.; Kozera, R.; Boczkowska, A. Poly (urea-formaldehyde) microcapsules-synthesis and influence of stirring speed on capsules size. Polimery 2018, 63, 339-346. [CrossRef]

23. Safaei, F.; Khorasani, S.N.; Rahnama, H.; Neisiany, R.E.; Koochaki, M.S. Single microcapsules containing epoxy healing agent used for development in the fabrication of cost efficient self-healing epoxy coating. Prog. Org. Coat. 2018, 114, 40-46. [CrossRef]

24. Sadrabadi, T.E.; Allahkaram, S.R.; Staab, T.; Towhidi, N. Preparation and characterization of durable micro/nanocapsules for use in self-healing anticorrosive coatings. Polym. Sci. Ser. B 2017, 59, 281-291. [CrossRef]

25. Comlekci, G.K.; Ulutan, S. Encapsulation of linseed oil and linseed oil based on alkyd resin by urea formaldehyde shell for self-healing systems. Prog. Org. Coat. 2018, 121, 190-200. [CrossRef]

26. Fereidoon, A.; Ahangari, M.G.; Jahanshahi, M. Effect of nanoparticles on the morphology and thermal properties of self-healing poly(urea-formaldehyde) microcapsules. J. Polym. Res. 2013, 20, 1-8. [CrossRef]

(C) 2019 by the authors. Licensee MDPI, Basel, Switzerland. This article is an open access article distributed under the terms and conditions of the Creative Commons Attribution (CC BY) license (http://creativecommons.org/licenses/by/4.0/). 